\title{
Pharmacists' knowledge attitude and practice regarding the dispensing of antibiotics without prescription in Tanzania: an explorative cross- sectional study
}

\section{Baraka P Poyongo}

Muhimbili University of Health and Allied Sciences

Raphael Zozimus Sangeda ( $\sim$ sangeda@gmail.com )

Muhimbili University of Health and Allied Sciences https://orcid.org/0000-0002-6574-5308

\section{Research}

Keywords: Antibiotics, antibiotics resistance, antimicrobial resistance, dispensing, pharmacist, prescription, Tanzania.

Posted Date: November 18th, 2019

DOl: https://doi.org/10.21203/rs.2.17343/v1

License: (c) (i) This work is licensed under a Creative Commons Attribution 4.0 International License.

Read Full License 


\section{Abstract}

Background: Inappropriate use of antibiotics has been reported to contribute to the emergence and increase of antimicrobial resistance (AMR) in the world. Enforcing the dispensing of antibiotics with prescription is a way to promote the rational use of antibiotics and preventing the development and spread of AMR. The pharmacist has the responsibility to supervise the dispensing of antibiotics in pharmacies and ensure its rational use. This study assessed pharmacists' knowledge, attitude and practice regarding the dispensing of antibiotics without prescription in Tanzania. Methods: An online semi-structured questionnaire was designed, tested and shared with licensed pharmacists in Tanzania through an invitation link sent in their official WhatsApp groups. An SMS alternative was used contact list from the Pharmacy Council to administer the questionnaire, to pharmacist not subscribed on WhatsApp. Study data were collected and managed using REDCap electronic data capture tools hosted at Muhimbili University of Health and Allied Sciences, Dar es Salaam, Tanzania. Data were then downloaded and exported into Statistical Package for Social Sciences (SPSS) version 20 for data analysis; Chi-square test was used to test association for categorical data, where a p-value of less than 0.05 was considered statistically significant. Results: More than $75 \%$ of pharmacists had excellent knowledge about the legal requirements for dispensing antibiotics and of the AMR challenge. Of the interviewed pharmacists, seventy-four percent admitted to dispensing antibiotics without prescription in their daily practice. Notably, $85.7 \%$ of pharmacists with high education were more likely to dispense without prescription compared to $69.9 \%$ among bachelor holders ( $p$-value $=0.028$ ). The main reasons for administering antibiotics without prescription were the profitability nature of pharmacy business, a failure of the patient to get a prescription and lack of stringent regulatory authorities. Penicillins, macrolides and fluoroquinolones were the classes of antibiotics mostly dispensed without a prescription. Conclusion: Even though pharmacists seem knowledgeable about AMR, they still dispense antibiotics without prescription in Tanzania. The regulatory authorities should make regular inspections in pharmacies to detect this malpractice. The community should be trained on the importance of taking laboratory tests before getting medications for their sickness by a qualified medical practitioner.

\section{Background}

Antimicrobial resistance (AMR) is the resistance of microorganisms to a drug that was once effective in treating infections caused by the microorganism[1]. Both appropriate and inappropriate use of antimicrobial agents leads to AMR development. However, inappropriate use of antimicrobial such as underuse, overuse or misuse of antimicrobial are the leading causes for the development of AMR and are preventable[2]. AMR has many consequences to both individual patients and the public at large, such as increasing cost burden of treatment, poor treatment outcome, and spread of resistant microbial to other people hence endangering the public health[3,4].

In many countries in the world, including Tanzania, antibiotics are prescription-only medicine meant to be dispensed only to a person with a prescription from an authorized physician; this promotes the appropriate use of antibiotics hence reducing the rate of AMR[5]. World Health Organization (WHO) urges 
that the fight against AMR and antibiotic resistance should involve collaboration among the national experts to understand all aspects of antibiotic access, use and resistance within their country context. These experts then work on drafting and implementing policy solutions tailored to meet the country's needs[6].

AMR poses a significant threat to clinical efficacy, especially in developing countries because they lack comprehensive measures to combat AMR[7]. There are growing concerns that pharmacists and other drug dispensers do not adhere to Good Dispensing Practises (GDP), especially in lower and middleincome countries and thus contributing to AMR development[8]. Some of the factors that contribute to AMR include the irrational use of antibiotics while some of the intrinsic changes of bacteria make them naturally resistant to antibiotics [9].

Antibiotics are antimicrobial agents that are used in the treatment of bacterial infections by either killing or inhibiting the growth of bacteria. Licensed pharmacists in community pharmacies are the ones responsible for the dispensing of antibiotics[10]. Dispensing of antibiotics without prescription in community pharmacies is one of the contributing factors for the irrational use of antibiotics, thus enhancing the emergence and spread of antibiotic resistance.

A couple of surveys have been conducted in Tanzania to evaluate dispensing practices of antibiotics in community pharmacies, Accredited Drug Dispensing Outlets (ADDOs) and Duka La dawa Baridi (DLDB) and other shops. The surveys revealed antibiotics dispensed without a prescription making apparent bleach of GDP. For instance, in a study conducted in Moshi municipality, in Kilimanjaro, Tanzania, 92.3\% of pharmacy dispensers issued antibiotics without a prescription[11]. Although there are regulatory authorities like the Tanzania Medicines and Medical Devices Authorities (TMDA) and the Pharmacy Council of Tanzania (PC) who supervise ADDO and Pharmacy shops, there are still reports of dispensing mal-practice in ADDOs and DLDB's. The cross-section survey done in four different districts in Tanzania found that dispensers were issuing antibiotics without prescription in ADDO shops and DLDBs[12]. Private rural drugstores do not adhere to GDP in dispensing antibiotics, people are issued antibiotics without a prescription after complaining to dispenser about their sickness like cough, flu and diarrhoea[13]. Tanzania regulations require a community pharmacy to be under the supervision of a licensed pharmacist. The pharmacist is responsible for making sure that the GDP protocols are adhered to during dispensing of drugs. A previous study conducted in Tanzania has shown that pharmacies and ADDOs dispense antibiotics without prescription. These studies investigated whether medicine dispensers in community pharmacies or ADDOs dispense without prescription regardless of their cadre, which could be a licensed pharmacist, pharmaceutical technician or pharmaceutical assistant. Since pharmacists are entitled to supervise the dispensing practice in pharmacies, their knowledge, attitude and practice are of concern as it may contribute to the irrational use of antibiotics and AMR development.

There is a lack of studies to evaluate pharmacists' knowledge, attitude and practice regarding the dispensing of antibiotics without prescription in Tanzania. Therefore, this study aimed to determine the 
proportion of licensed pharmacists who dispense without prescription, reasons for dispensing antibiotics without prescription and their awareness of the legal requirements to dispense antibiotics and AMR.

The findings of this study may be useful to the Government and regulatory authorities like the Pharmacy Council of Tanzania in advising mechanisms and measures to be taken in combating AMR.

\section{Methods}

\section{Study site}

The study was conducted in all regions of Tanzania, a country found in East Africa. In 2019, Tanzania is estimated to have a population of 55 million (approximation based on the 2012 national census)[14] and a total of 1,863 licensed pharmacists[15].

\section{Study design and Sampling}

The study was a descriptive cross-sectional conducted from January 2019 to July 2019 . The sample size was calculated based on previous research with a prevalence of dispensing antibiotics without prescription of $80 \%$ [9], for $95 \%$ confidence level and a $5 \%$ margin of error, resulted in a sample size of 245.

\section{Study tools}

An online semi-structured questionnaire with both open and mostly closed-ended questions was designed and pre-tested. An invitation link was shared with pharmacists through WhatsApp groups where most of the pharmacists participate. Most licensed pharmacists across the country were invited to participate in this manner. Besides, a list of names, contacts and emails of licensed pharmacists obtained from the Pharmacy Council of Tanzania and used to contact and request pharmacists to fill the questionnaire directly. A REDCap (Research Electronic Data Capture) survey was created for online data collection of pharmacists' responses.

Data were collected and managed using REDCap; electronic data capture tools hosted at Muhimbili University of Health and Allied Science. REDCap is a secure, web-based application designed to support data capture for research studies, providing 1 ) an intuitive interface for validated data entry; 2) audit trails for tracking data manipulation and export procedures; 3 ) automated export procedures for seamless data downloads to standard statistical packages; and 4) procedures for importing data from external sources[16,17]

The questionnaire had five sections; the first section was for obtaining social-demographic characteristics of respondents such as gender, age, level of education, country of graduation of bachelor of pharmacy, job status in community pharmacy, region of practice and experience. The second section aimed to evaluate pharmacists' knowledge of legal needs to dispense antibiotics, AMR and to assess pharmacists' attitudes towards dispensing of antibiotics without prescription. Questions to investigate if 
pharmacists were aware that the dispensing of antibiotics without prescription is an illegal practice in Tanzania and can lead to the emergence and spread of AMR were asked. Furthermore, they were probed on what they think of any problem with dispensing without prescription and whether pharmacists should stop this practice.

The third section of the questionnaire probed out the reasons that influence the dispensing of antibiotics without prescription. These reasons were divided into two categories, which are patients based and pharmacists based reasons. Patient based factors that drive a patient to visit a pharmacy without a prescription when they are sick. Such factors may be the fear of patients to use many hours of waiting in a hospital, lack of insurance cover, patient, do not want to see a doctor unless the disease is severe, patients unable to afford the cost for consultation and laboratory tests. Any other reasons were provided by the pharmacists selecting a factor that mostly influence patients to visit pharmacy without a prescription. Pharmacists based reasons for dispensing without prescription, including the profit-oriented nature of pharmacy business, the relationship between pharmacist and patient, and the belief that pharmacists are knowledgeable enough to dispense without prescription were evaluated. Additionally, we assessed the influence of regular inspection of pharmacies by regulatory authorities on dispensing without prescription.

The fourth section investigated the classes of antibiotics, mostly dispensed without a prescription. Besides, the medical conditions to which antibiotics are mostly dispensed without prescription were investigated. Pharmacists were asked to select a class of antibiotics from the list that they would easily dispense to a patient without a prescription suffering from suspected bacterial infection. They were also asked to select from the list two medical conditions to which antibiotics are administered without prescription based on their practice and experience.

The fifth section assessed pharmacists' practice and determine the proportion of pharmacists who dispense antibiotics without prescription in Tanzania. Pharmacists were questioned if they would dispense antibiotics to a person without a prescription. Pharmacists were also asked if they do inform a patient about, side effects of antibiotics, drug-drug/food interactions and the importance of adherence to the dispensed course of antibiotics.

\section{Statistical analysis}

Data were downloaded from REDCap, cleaned and analysed using Statistical Package for Social Sciences (SPSS) version 20, a total of 213 pharmacists completed the questionnaire. Descriptive statistical analysis was performed and a Chi-square test for categorical data was used where a p-value of less than 0.05 was considered to be statistically significant.

\section{Ethical considerations}

Ethical clearance to conduct this study was obtained from Muhimbili University of Health and Allied Sciences Research and Publications Committee with reference number DA.25/111/01. Written consent 
was obtained before filling the questionnaire after a thorough explanation of the study objectives and a pharmacist was free to reject participating in the study. The pharmacist's name was not recorded (only codes were used) and all other personal information was handled with confidentiality throughout the research and during presentations.

\section{Results}

\section{Socio-demographic characteristics}

A total of 1,100 licensed pharmacists were contacted and requested to fill the questionnaire through an online invitation link. A total of 226 (20.5\%) responses were recorded, of which 213 responses were complete and used for analysis. Out of 213 respondents, 148 (69.5\%) were males and 65 (30.5\%) were females.

The highest education level for most of the respondents was a bachelor degree in pharmacy 164 (77.0\%), $46(21.6 \%)$ had masters and only $3(1.4 \%)$ respondents were PhD holders. Out of 211 pharmacists, 195 (92.4\%) pharmacists completed their bachelor studies in Tanzania, 9 (4.3\%) in India and the rest 7 (3.3\%) from other countries.

Out of 211 respondents, 134 (63.5\%) work in community pharmacies where 14 (10.4\%) were owners, 89 (66.4\%) were dispensing pharmacist and 31 (23.2\%) were both owner and dispensing pharmacists. The majority, 68 (51.9\%) of the respondents work in community pharmacies that are located in Dar es Salaam region, 12 (9.2\%) in Mwanza while Arusha region, Mara region, Mbeya region and Tanga region each contributed 5 (3.8\%) of responses from community pharmacies in respective regions. The summary of social demographic data of the respondents is in Table 1.

\section{Pharmacists' knowledge}

Ten questions covering legal requirements for dispensing antibiotics and knowledge of AMR were asked and scored. The items assessed if a pharmacist was aware of different rules, regulations and laws that govern the dispensing of antibiotics. The questions also were designed to evaluate if pharmacists know about AMR as one of the consequences of dispensing antibiotics without prescription. Each correct answer was given a score of 10 points, after which an overall score was calculated per respondent. The maximum score was 100 points, while the minimum score was 20 points. A Likert scale was used to categorize knowledge whereby those who scored 80-100 points were labelled as excellent knowledge, 6070 points were labelled very good knowledge, 40-50 points were labelled good knowledge while those who scored 20-30 points were regarded to have poor knowledge and 0-10 points were labelled very poor knowledge (Figure 1).

The responses of pharmacists to questions assessing knowledge were as follows: $79.7 \%$ of respondents were aware that the dispensing of antibiotics without prescription is illegal in Tanzania and $20.3 \%$ did not know that the practice is illegal. 114 (53.8\%) pharmacists were not sure if there is a penalty to a person 
who dispenses without prescription. More than $90.0 \%$ of pharmacists agreed that dispensing without prescription contributes to the irrational use of antibiotics and the development of AMR. $75.1 \%$ of pharmacists had overall excellent knowledge, $17.4 \%$ very good knowledge and $3.3 \%$ poor knowledge (Figure 1).

\section{Pharmacists' attitude towards dispensing of antibiotics without prescription}

Five questions were asked to assess pharmacists' attitudes on dispensing antibiotics without prescription. Each response was scored 10 for the positive attitude and 0 scores for a negative attitude. The overall score was recorded and those who scored 30-50 out of 50 were labelled to have positive attitude while those who scored less than 30 out of 50 were considered to have a negative attitude on dispensing antibiotics without prescription.

Concerning pharmacists' attitude, 38.8\% (82) had a negative attitude while 61.2\% (129) pharmacists had a positive attitude on dispensing antibiotics without prescription. A positive attitude was for the ones who thought dispensing without prescription should be stopped as it has negative consequences to the patient and society in general, and other related questions, as shown in table 2.

\section{Pharmacists practice}

Out of 212 pharmacists, $156(74 \%)$ agreed that they do dispense antibiotics to a patient without a prescription in their practice and $56(26 \%)$ denied to dispensed antibiotics without prescription. A large proportion of pharmacists who work in community pharmacies dispense without prescription more than those who do not work in community pharmacies $(p<0.001)$. Education level was observed to influence the practice as $85.7 \%$ (42/49) pharmacists with high education (masters and PhD holder) admitted to dispensing without prescription compared to $69.9 \%$ (114/163) bachelor holder who dispenses without prescription (chi-square $\mathrm{p}$-value of 0.028 ). This suggests that with the increase in the education level, the more a pharmacist feels confident to dispense without prescription. There was a strong association between pharmacists' attitudes and dispensing without prescription $(p<0.001)$. Age $(p=0.651)$, experience $(p=0.707)$ and country of graduation $(p=0.394)$ had no statistical association with the dispensing of antibiotics without prescription. More than $60.0 \%$ of pharmacists claimed to counsel their customers about drug-drug/food interaction and insist patients adhere to the dispensed course of medication.

Out of the 156 pharmacists who admitted to dispensing antibiotics without prescription, 95 (60.9\%) promised to stop this practice in the future. They felt this was a bad practice that contributes to the irrational use of antibiotics and has the potential to influence the development and spread of AMR. 57 (36.5\%) pharmacists denied to stop the practice even in the future as they regarded the practice to be helpful to the patient and pharmacists being knowledgeable can help a patient to get the right antibiotics even without laboratory tests and a prescription. Some of the reasons which were given out by pharmacists for stopping and not stopping dispensing antibiotics without prescription in the future are summarized in table 3. 
Reasons for dispensing without prescription were also revealed. Some of the reasons were attributed to pharmacists to dispense without prescription, included pharmacists thinking that they are knowledgeable enough to dispense without prescription 150 (71.1\%), pharmacists being in close relationship with a customer who needs antibiotics 106 (50.5\%) and business nature of pharmacy that focuses on making more profit. Out of 210 pharmacists, 140 (67\%) think that pharmacists will stop dispensing antibiotics without prescription if there could be a regular inspection by regulatory authorities like the Pharmacy Council and the TMDA.

Patients based reasons included that push patients to visit pharmacies without a prescription asking for antibiotics when they are sick were noted. The leading causes were the fear of spending many hours during the patients' visit to the health facilities and wait for a consultation to complete (79.0\%). The next leading cause was the inability of patients to afford consultation fee and cost for a laboratory test. in Tanzania. The light-blue colour portion agreed to dispense antibiotics without prescription, while the red part denied.

\section{Classes of antibiotics that are mostly dispensed without a prescription}

Pharmacists responded to the question that asked what antibiotics would they readily and easily dispense to a patient without a prescription suffering from suspected bacterial infection by selecting from the list. A respondent was allowed to choose more than one class of antibiotics that he/she will feel comfortable to dispense to a patient who has no prescription.

Regarding the type of antibiotics, the pharmacist would readily dispense to a patient suffering from suspected bacterial infection without a prescription. Penicillins 169 (79.3\%) was the most selected class of antibiotics, followed by macrolides 86 (40.3) and fluoroquinolones 69 (32.4\%) (Figure 3).

\section{Medical conditions to which antibiotics are commonly administered without a prescription}

Pharmacists mentioned some diseases to which antibiotics are usually dispensed without a prescription. The leading conditions were urinary tract infection 149 (70.0\%), cough $115(54.0 \%)$ and sexually transmitted diseases 87 (40.8\%) (Figure 4).

\section{Discussion}

Inappropriate dispensing of antibiotics has been reported to be a common practice in many developing countries[18]. A simulated study conducted in one of the regions in Tanzania reported more than $90 \%$ of part I and part II pharmacies to dispense antibiotics without prescription[11]. Another cross-sectional explorative study conducted in Morogoro and Pwani region in Tanzania that observed dispensing practice in ADDOs reported that the shops do not follow the dispensing regulations of antibiotics[12]. To our understanding, this is the first study to evaluate pharmacists' knowledge, attitude and practice regarding the dispensing of antibiotics without prescription in Tanzania. 
About $80 \%$ of pharmacists were aware that dispensing without prescription is illegal in Tanzania contrary to a study conducted in Saudi Arabia, where only a quarter of pharmacist was aware[9]. Despite the majority of pharmacists having excellent knowledge about legal requirements to dispense antibiotics and consequences of the irrational use of antibiotics, $74.0 \%$ pharmacists agreed that they dispense without prescription in their practice. These results were considered similar to a study conducted in Syria where 89.3\% pharmacists admitted to dispensing antibiotics without prescription[7] while a simulated research conducted in India and Kilimanjaro region in Tanzania revealed that $66.7 \%$ and $90.0 \%$ pharmacies dispense antibiotics without prescription respectively[11,19]

Patients, pharmacists and the regulatory authorities' practices may all contribute to this mal-practice. The tendency of patients to visit pharmacy without a legal prescription was claimed by pharmacists to be among the reason for them to dispense without prescription to meet customer demands and expectations. Fear to use many hours when they visit hospital and inability to afford consultation fee and laboratory tests cost were the leading factors that hinder the patient from obtaining a prescription. Similar reasons were revealed in a study conducted in Saudi Arabia and in Khartoum Sudan[5,9]. Pharmacists seem to dispense antibiotics without prescription to maximize sales and profit because they think the regulatory authorities are not stringent enough to prevent inappropriate dispensing of antibiotics. If a patient is denied antibiotics she/he will obtain it in other pharmacies, as one pharmacist responded "if they don't get it from my pharmacy they will get it from another pharmacy", the same reasons were reported in other study conducted in ADDOs in Tanzania that evaluated the reasons that motivate dispensers in ADDOs to dispense antibiotics without prescription[20].

Self believes that pharmacists are knowledgeable enough about diseases and antibiotics was a reason for malpractice as pharmacists who were masters and PhD holders dispensed without prescription more than bachelor holder, this shows that as the level of education increase a pharmacist confidence increase and thinks that he/she has a knowledge enough to evaluate a patient sickness and treat accordingly even giving antibiotics without prescription. Some of the responses of pharmacists to why they dispense without prescription were such as: "some conditions are so obvious that you really know the patient sickness", "pharmacists are drug experts hence can make good choice of antibiotics if he or she is sure of the condition", "I have the knowledge that will clinically guide me to dispense when there are a need and the infections is not that much serious". Some pharmacists claimed that there is a Pharmacist Initiated Therapy (PIT) that allows them to dispense without prescription. This was a misconception of the PIT where only non-prescription drugs are allowed to be prescribed and dispensed by a pharmacist without a prescription [21].

Antibiotics from penicillins and macrolides classes were found to be the most dispensed antibiotics. These findings are similar to other surveys conducted in Ethiopia and Egypt[22,23] and a little bit different from a study conducted in Saudi Arabia where penicillins and cephalosporins were the mostly dispensed antibiotics without prescription[9]. The observed difference may be due to the variability of the prevalence of diseases in the two countries as urinary tract infections and cough were the common medical conditions to which antibiotics are mostly dispensed without prescription in Tanzania while in Saudi 
Arabia it was cold and flu, rhinitis and toothache[9]. Irrational use keeps penicillins and macrolides at a high risk of resistance, since the resistant bacteria are more likely to emerge with inappropriate use of these essential classes of antibiotics. This further complicates the future treatment choices for bacterial infections. WHO has already reported the resistance of penicillins and fluoroquinolones in several countries[24].

Preventing inappropriate use of antibiotics and the growth of AMR is the goal of any health sector in any country. AMR is caused by interconnected factors involving patients, health care providers and regulatory authorities. Coordinated interventions at both national and international levels are required in combating the AMR. Creating awareness about AMR in society will be a first step in promoting the rational use of antibiotics as proposed in a national action plan on antibiotic resistance[25]. From the findings of this study, the government should consider decreasing the consultation fee and laboratory test costs as these were the main hindrance for patients to get a prescription. Strict regulatory authorities and regular inspections are the core for tackling the dispensing of antibiotics without prescription in pharmacies. In countries where there are strict functioning regulatory authorities, the mal-practices in pharmacies has been observed to decline[26,27]

There are some limitations to this study, pharmacists were invited to fill the online questionnaire via an invitation link and there was no physical contact between interviewer and interviewee, however, we are certainly sure that pharmacists are the ones who filled the questionnaire because the link was shared only to pharmacists after obtaining their contacts from pharmacy council the board responsible for control and management of the pharmacy profession in Tanzania. Another limitation is that all licensed pharmacists were included in the study regardless of working in the community pharmacies or not, this was done to know the attitude of pharmacists and their remarks regarding dispensing of antibiotics without prescription. The majority of respondents were from Dar es Salaam, a city with the highest population in Tanzania and few respondents from other regions of the country. More than $50 \%$ of community pharmacies are located in Dar es Salaam and the rest in other upcountry regions, making the findings of this study to be valid to represent the whole country.

\section{Conclusion}

The Tanzanian pharmacists seem to be knowledgeable of the legal requirements to dispense antibiotics, the antimicrobial resistance threat and its consequences to both patient and public at large. However, the majority of these pharmacists admitted to dispensing antibiotics without prescription and claimed that the practice is common in numerous community pharmacies in Tanzania. The business nature of pharmacy, a failure of the patient to get a prescription and the lack of rigorous regulatory authorities' actions were cited as the main reasons for this violation. The antibiotics frequently reported to be dispensed without a prescription were penicillins, macrolides and fluoroquinolones. These antibiotics were used for the treatment of medical conditions such as urinary tract infections, cough and sexually transmitted diseases. 
There is an obvious need for educating the community regarding antimicrobial resistance and the importance of visiting the nearby health centre for consultation and laboratory tests before obtaining medications. Community awareness will help in tackling the irrational use of antibiotics, emergence and spread of antimicrobial resistance. On one hand, the government should consider reducing the consultation fee and laboratory test costs in public health centres since these are a considerable barrier for the majority of patients to afford. On the other hand, the regulatory authorities should make regular inspections to pharmacies to detect any prevailing malpractice. In case a medicine dispenser is caught intentionally issuing prescription-only medicines without a prescription, the person should be penalized according to the current pharmacy profession laws in the country.

\section{List Of Abbreviations}

ADDOs: Accredited Drug Dispensing Outlets

AMR: Antimicrobial resistance

DLDB: Duka La Dawa Baridi (Swahili for essential medicine drug outlets)

GDP: Good Dispensing practice

PIT: Pharmacist Initiated Therapy

REDCap: Research Electronic Data Capture

TMDA: Tanzania Medicines and Medical Devices Authority

\section{Declarations}

\section{Ethics approval and consent to participate}

Ethical clearance to conduct this study was obtained from Muhimbili University of Health and Allied Sciences Research and Publications Committee with reference number DA.25/111/01. Written consent was obtained before filling the questionnaire after a thorough explanation of the study objectives and a pharmacist was free to reject participating in the study. The pharmacist's name was not recorded (only codes were used) and all other personal information was handled with confidentiality throughout the research and during presentations.

\section{Consent for publication}

Not applicable

\section{Availability of data and material}

The dataset analysed during the current study is available from the corresponding author on request. 


\section{Funding}

The study was self-funded by authors.

\section{Authors' contributions}

Both of the co-authors contributed sufficiently in the manuscript as follows, BPP contributed to the study design, data collection, analysis and writing the manuscript. RZS contributed to the design of the study and review of the manuscript. Both authors have read and approved the final manuscript.

\section{Acknowledgments}

We wish to express our sincere gratitude to all the pharmacists who participated in the study.

\section{Competing interest}

The authors declare that they have no competing interests.

\section{Authors' details}

Baraka P Poyongo ${ }^{1}$, barakapoyongo@gmail.com

Raphael Z Sangeda*, sangeda@gmail.com

${ }^{1}$ Department of Pharmaceutical Microbiology, Muhimbili University of Health and Allied Sciences, Dar es Salaam, Tanzania

* Corresponding author

\section{References}

1. Levy SB, Marshall B. Antibacterial resistance worldwide: causes, challenges and responses. Nat Med. 2004;10:S122-9.

2. Nga DTT, Chuc NTK, Hoa NP, Hoa NQ, Nguyen NTT, Loan HT, et al. Antibiotic sales in rural and urban pharmacies in northern Vietnam: an observational study. BMC Pharmacol Toxicol. 2014;15:6.

3. Stewardson AJ, Allignol A, Beyersmann J, Graves N, Schumacher M, Meyer R, et al. The health and economic burden of bloodstream infections caused by antimicrobial-susceptible and nonsusceptible Enterobacteriaceae and Staphylococcus aureus in European hospitals, 2010 and 2011: a multicentre retrospective cohort study. Eurosurveillance. 2016;21:30319.

4. de Kraker MEA, Davey PG, Grundmann H. Mortality and Hospital Stay Associated with Resistant Staphylococcus aureus and Escherichia coli Bacteremia: Estimating the Burden of Antibiotic Resistance in Europe. Opal SM, editor. PLoS Med. 2011;8:e1001104. 
5. Salim AMA, Elgizoli B. Exploring the reasons why pharmacists dispense antibiotics without prescriptions in Khartoum state, Sudan. Int J Pharm Pract. 2017;25:59-65.

6. Leung E, Weil DE, Raviglione M, Nakatani $\mathrm{H}$. The WHO policy package to combat antimicrobial resistance. Bull World Health Organ. 2011;89:390-2.

7. Bahnassi A. A qualitative analysis of pharmacists' attitudes and practices regarding the sale of antibiotics without prescription in Syria. J Taibah Univ Med Sci. 2015;10:227-33.

8. Ayukekbong JA, Ntemgwa M, Atabe AN. The threat of antimicrobial resistance in developing countries: causes and control strategies. Antimicrob Resist Infect Control. 2017;6:47.

9. Hadi MA, Karami NA, Al-Muwalid AS, Al-Otabi A, Al-Subahi E, Bamomen A, et al. Community pharmacists' knowledge, attitude, and practices towards dispensing antibiotics without prescription (DAwP): a cross-sectional survey in Makkah Province, Saudi Arabia. Int J Infect Dis. Elsevier; 2016;47:95-100.

10. Vazquez-Lago J, Gonzalez-Gonzalez C, Zapata-Cachafeiro M, Lopez-Vazquez P, Taracido M, López A, et al. Knowledge, attitudes, perceptions and habits towards antibiotics dispensed without medical prescription: A qualitative study of Spanish pharmacists. BMJ Open. 2017;7.

11. Horumpende PG, Sonda TB, van Zwetselaar M, Antony ML, Tenu FF, Mwanziva CE, et al. Prescription and non-prescription antibiotic dispensing practices in part I and part II pharmacies in Moshi Municipality, Kilimanjaro Region in Tanzania: A simulated clients approach. PLoS One. Public Library of Science; 2018;13.

12. Minzi O, Manyilizu. Application of basic pharmacology and dispensing practice of antibiotics in accredited drug-dispensing outlets in Tanzania. Drug Healthc Patient Saf. 2013;5:5.

13. Viberg N, Kalala W, Mujinja P, Tomson G, Lundborg CS. "Practical knowledge" and perceptions of antibiotics and antibiotic resistance among drugsellers in Tanzanian private drugstores. BMC Infect Dis. 2010;10:270.

14. National Bureau of Statistics. Report of 2012 Population and Housing census, The United Republic of Tanzania [Internet]. 2013 [cited 2019 Nov 6]. p. 65. Available from:

https://www.nbs.go.tz/nbs/takwimu/census2012/Basic_Demographic_and_SocioEconomic_Profile_PopularVersion-KeyFindings_2012_PHC_EnglishVersion.pdf

15. The Pharmacy Council of Tanzania. Pharmacy council Tanzania newsletter [Internet]. 2019 [cited 2019 Nov 6]. Available from: https://www.pc.go.tz/files/Pharmacy Council Newsletter April - June 2019.pdf

16. Harris PA, Taylor R, Thielke R, Payne J, Gonzalez N, Conde JG. Research electronic data capture (REDCap)-a metadata-driven methodology and workflow process for providing translational research informatics support. J Biomed Inform. 2009;42:377-81.

17. Harris PA, Taylor R, Minor BL, Elliott V, Fernandez M, O’Neal L, et al. The REDCap consortium: Building an international community of software platform partners. J Biomed Inform. Academic Press; 2019;95:103208. 
18. Erku DA, Aberra SY. Non-prescribed sale of antibiotics for acute childhood diarrhea and upper respiratory tract infection in community pharmacies: a 2 phase mixed-methods study. Antimicrob Resist Infect Control. 2018;7:92.

19. Shet A, Sundaresan S, Forsberg BC. Pharmacy-based dispensing of antimicrobial agents without prescription in India: appropriateness and cost burden in the private sector. Antimicrob Resist Infect Control. 2015;4:55.

20. Dillip A, Embrey M, Shekalaghe E, Ross-Degnan D, Vialle-Valentin C, Kimatta S, et al. What motivates antibiotic dispensing in accredited drug dispensing outlets in Tanzania? A qualitative study. Antimicrob Resist Infect Control. 2015;4:30.

21. Dekker A, Smit R. Pharmacist initiated therapy: recognition and treatment of minor ailments. Juta and Company Ltd; 1993.

22. Gebretekle GB, Serbessa MK. Exploration of over the counter sales of antibiotics in community pharmacies of Addis Ababa, Ethiopia: pharmacy professionals' perspective. Antimicrob Resist Infect Control. 2016;5:2.

23. Dooling K, Kandeel A, Hicks L, El-Shoubary W, Fawzi K, Kandeel Y, et al. Understanding Antibiotic Use in Minya District, Egypt: Physician and Pharmacist Prescribing and the Factors Influencing Their Practices. Antibiotics. 2014;3:233-43.

24. Yewale VN. Antimicrobial resistance - A ticking bomb! Indian Pediatr. 2014;51:171-2.

25. United Republic of Tanzania. Tanzania National Antimicrobial Resistance Action Plan [Internet]. 2017 [cited 2019 Nov 6]. Available from: https://www.flemingfund.org/wpcontent/uploads/8b8fc897c422e11504c8c2ba126fac02.pdf

26. Akinyandenu $O$, Akinyandenu A. Irrational use and non-prescription sale of antibiotics in Nigeria: $A$ need for change. J Sci Innov Res. 2014;3:251-7.

27. Bavestrello L, Cabello A, Casanova D. Impact of regulatory measures in the trends of community consumption of antibiotics in Chile. Rev Med Chil. 2002;130:1265-72.

\section{Tables}

Table 1: Social-demographic data of Tanzanian pharmacists responding to the questionnaire 


\begin{tabular}{|c|c|c|c|c|}
\hline \multicolumn{2}{|l|}{ Parameter } & frequency & percent & Percentiles \\
\hline \multirow[t]{3}{*}{ Age $(n=211)$} & $21-30$ & 101 & 47.9 & \multirow[t]{3}{*}{$28(31) 39$} \\
\hline & $31-40$ & 76 & 36.0 & \\
\hline & $>40$ & 34 & 16.1 & \\
\hline \multirow[t]{2}{*}{ Experience, years $(n=206)$} & $1-5$ & 132 & 64.1 & \multirow[t]{2}{*}{$2(4) 9$} \\
\hline & $>5$ & 74 & 35.9 & \\
\hline \multicolumn{2}{|l|}{$\operatorname{Sex}(n=213)$} & & & \\
\hline \multicolumn{2}{|l|}{ Male } & 148 & 69.5 & \\
\hline \multicolumn{2}{|l|}{ Female } & 65 & 30.5 & \\
\hline \multicolumn{2}{|l|}{ Total } & 213 & 100 & \\
\hline \multicolumn{4}{|l|}{ Education level $(\mathrm{n}=213)$} & \\
\hline \multicolumn{2}{|l|}{ Bachelor } & 164 & 77.0 & \\
\hline \multicolumn{2}{|l|}{ Masters } & 46 & 21.6 & \\
\hline \multicolumn{2}{|l|}{$\mathrm{PhD}$} & 3 & 1.4 & \\
\hline \multicolumn{2}{|l|}{ Total } & 213 & 100 & \\
\hline \multicolumn{4}{|l|}{ Country of graduation $(n=211)$} & \\
\hline \multicolumn{2}{|l|}{ Tanzania } & 195 & 92.4 & \\
\hline \multicolumn{2}{|l|}{ India } & 9 & 4.3 & \\
\hline \multicolumn{2}{|l|}{ Other } & 7 & 3.3 & \\
\hline \multicolumn{2}{|l|}{ Total } & 211 & 100 & \\
\hline \multicolumn{5}{|c|}{ Work in any community Pharmacy? $(n=213)$} \\
\hline \multicolumn{2}{|l|}{ Yes } & 134 & 63.0 & \\
\hline \multicolumn{2}{|l|}{ No } & 79 & 37.0 & \\
\hline \multicolumn{2}{|l|}{ Total } & 213 & 100 & \\
\hline \multicolumn{4}{|l|}{ Job status in community pharmacy $(n=134)$} & \\
\hline \multicolumn{2}{|l|}{ Owner } & 14 & 10.5 & \\
\hline \multicolumn{2}{|l|}{ Dispensing pharmacists } & 89 & 66.4 & \\
\hline \multicolumn{2}{|l|}{ Both owner and dispensing pharmacist } & 31 & 23.1 & \\
\hline \multicolumn{2}{|c|}{ Total } & 134 & 100 & \\
\hline \multicolumn{4}{|c|}{$\begin{array}{l}\text { Region of the practice of community pharmacist } \\
\qquad(\mathrm{n}=134)\end{array}$} & \\
\hline Dar es Salaam & & 68 & 50.7 & \\
\hline Mwanza & & 12 & 9.0 & \\
\hline Tanga & & 5 & 3.7 & \\
\hline Arusha & & 5 & 3.7 & \\
\hline Mbeya & & 5 & 3.7 & \\
\hline
\end{tabular}




\begin{tabular}{|l|r|r|} 
Mara & 5 & 3.7 \\
\hline Other & 34 & 25.5 \\
\hline Total & $\mathbf{1 3 4}$ & $\mathbf{1 0 0}$ \\
\hline
\end{tabular}

Table 2: Pharmacists' attitudes towards dispensing antibiotics without prescription

\begin{tabular}{|l|l|l|}
\hline \multirow{2}{*}{$\begin{array}{l}\text { No you think there is any problem if you dispense antibiotics without } \\
\text { prescription? }\end{array}$} & $\begin{array}{l}\text { N=211 } \\
(94.3 \%)\end{array}$ & $\begin{array}{l}12 \\
(5.7 \%)\end{array}$ \\
\cline { 2 - 3 } & YES & NO \\
\hline Pharmacist should stop dispensing without prescription & $\begin{array}{l}166 \\
(78.7 \%)\end{array}$ & $\begin{array}{l}45 \\
(21.3 \%)\end{array}$ \\
\hline $\begin{array}{l}\text { I encourage the patient to consult a physician and get a prescription } \\
\text { before visiting the pharmacy }\end{array}$ & $\begin{array}{l}118 \\
(55.9 \%)\end{array}$ & $\begin{array}{l}93 \\
(44.1 \%)\end{array}$ \\
\hline $\begin{array}{l}\text { Pharmacists are knowledgeable enough to dispense without } \\
\text { prescription after critical evaluation of patient sickness }\end{array}$ & $\begin{array}{l}150 \\
(71.1 \%)\end{array}$ & $\begin{array}{l}61 \\
(28.9 \%)\end{array}$ \\
\hline $\begin{array}{l}\text { Do you dispense antibiotics without prescription? } \\
156 \\
(74.0 \%)\end{array}$ & $\begin{array}{l}55 \\
(26.0 \%)\end{array}$ \\
\hline
\end{tabular}

Table 3: Pharmacists' reasons for stopping or not stopping dispensing antibiotics without prescription

\begin{tabular}{|l|l|l|}
\hline Reasons for stopping dispensing antibiotics without prescription $(\mathbf{n}=\mathbf{8 3})$ & frequency & percent \\
\hline To prevent the development and spread of AMR & 46 & 55.4 \\
\hline To promote rational use of medicines & 13 & 15.6 \\
\hline There are stringent regulatory authorities & 11 & 13.2 \\
\hline The practice is illegal & 9 & 10.8 \\
\hline There will be adequate health facilities in the future & 4 & 5.0 \\
\hline Reasons for not stopping the practice in the future $(\mathbf{n}=\mathbf{5 7})$ & & \\
\hline Pharmacists are knowledgeable enough to dispense without a prescription & 29 & 50.9 \\
\hline Profitability nature of pharmacies business & 12 & 21.1 \\
\hline Not enough health facilities for patients to obtain a prescription & 9 & 15.8 \\
\hline No stringent regulatory authorities & 7 & 12.2 \\
\hline
\end{tabular}


Figures

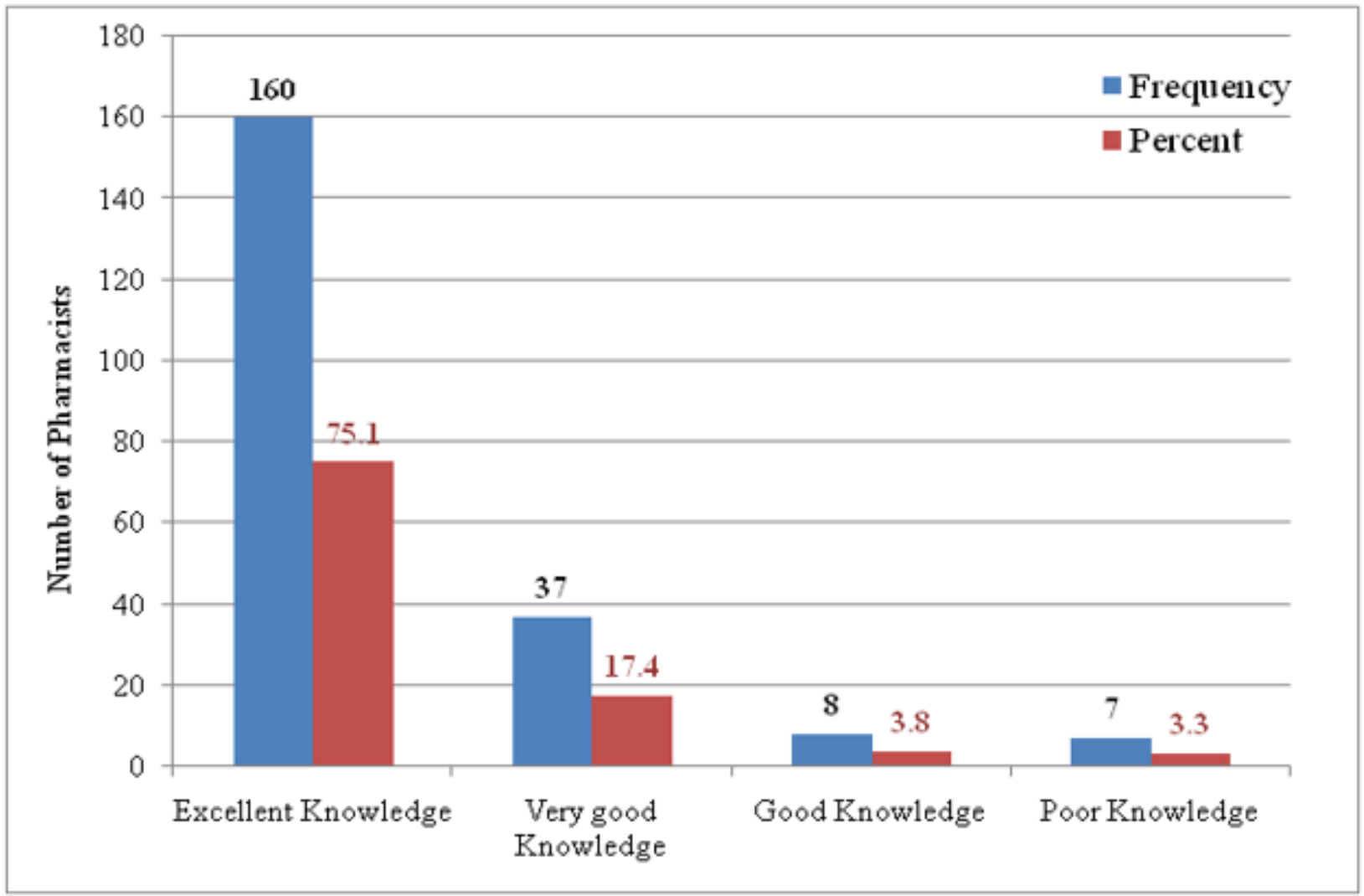

\section{Figure 1}

The overall pharmacist knowledge of legal needs to dispense antibiotics and AMR. The Likert scale was used to categorize pharmacists based on their scores on the ten questions asked to assess pharmacist knowledge. 
- Dispense without prescription $\quad$ Dispense with prescription

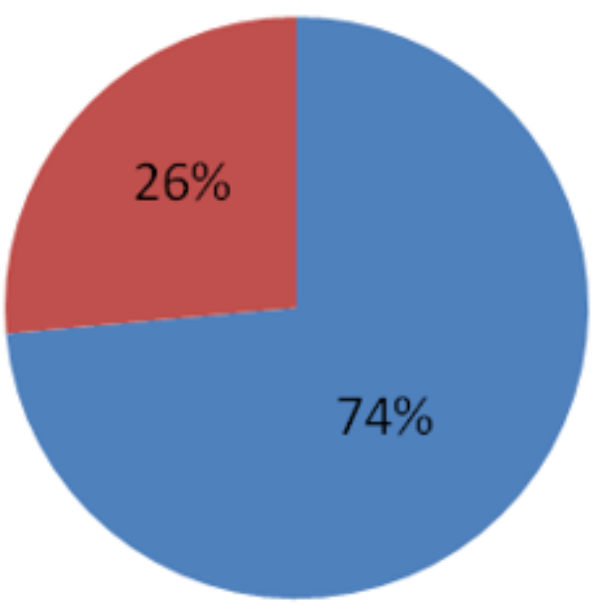

Figure 2

Proportion of licensed pharmacists who dispense antibiotics without prescription

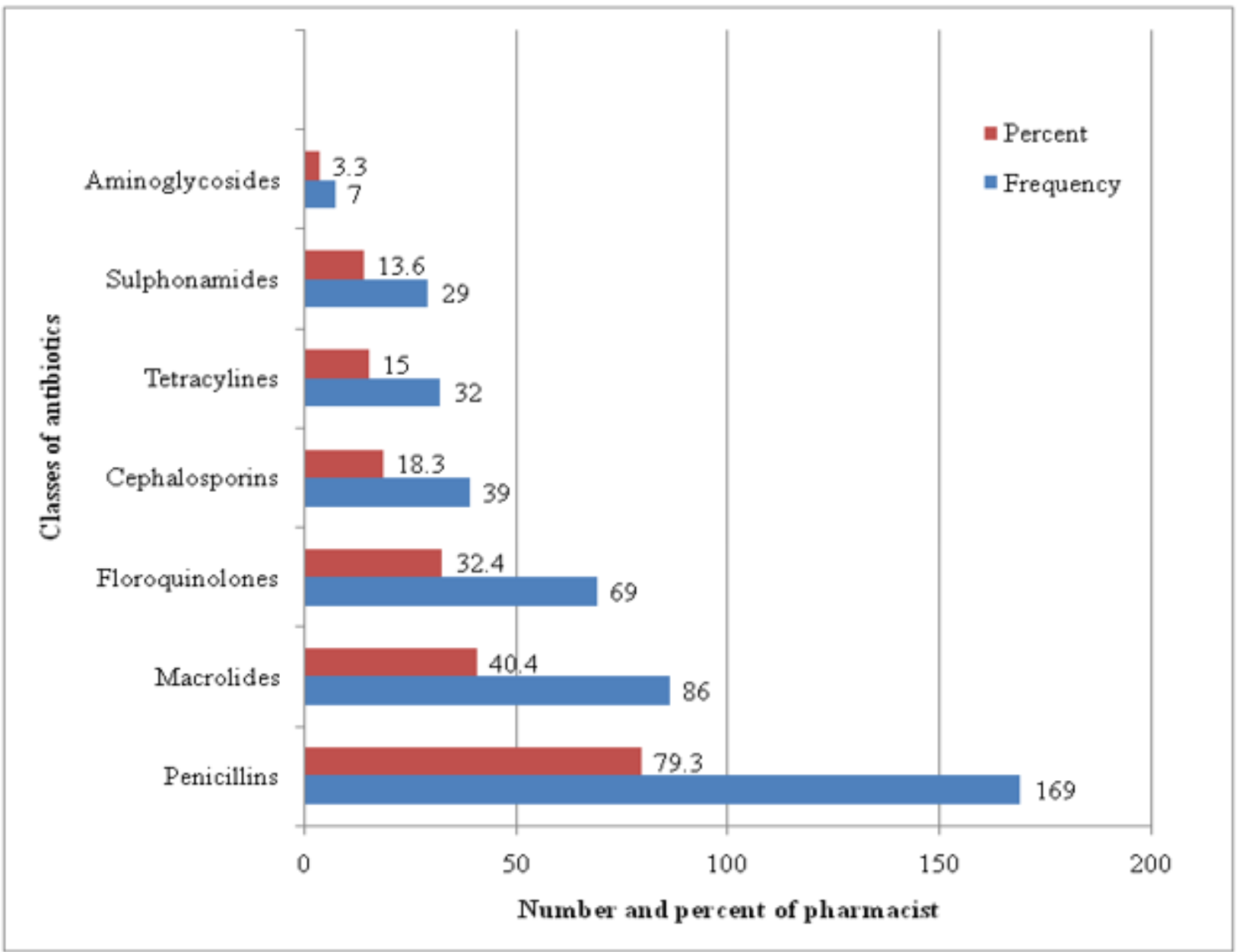


Figure 3

Class of antibiotics which mostly dispensed without a prescription

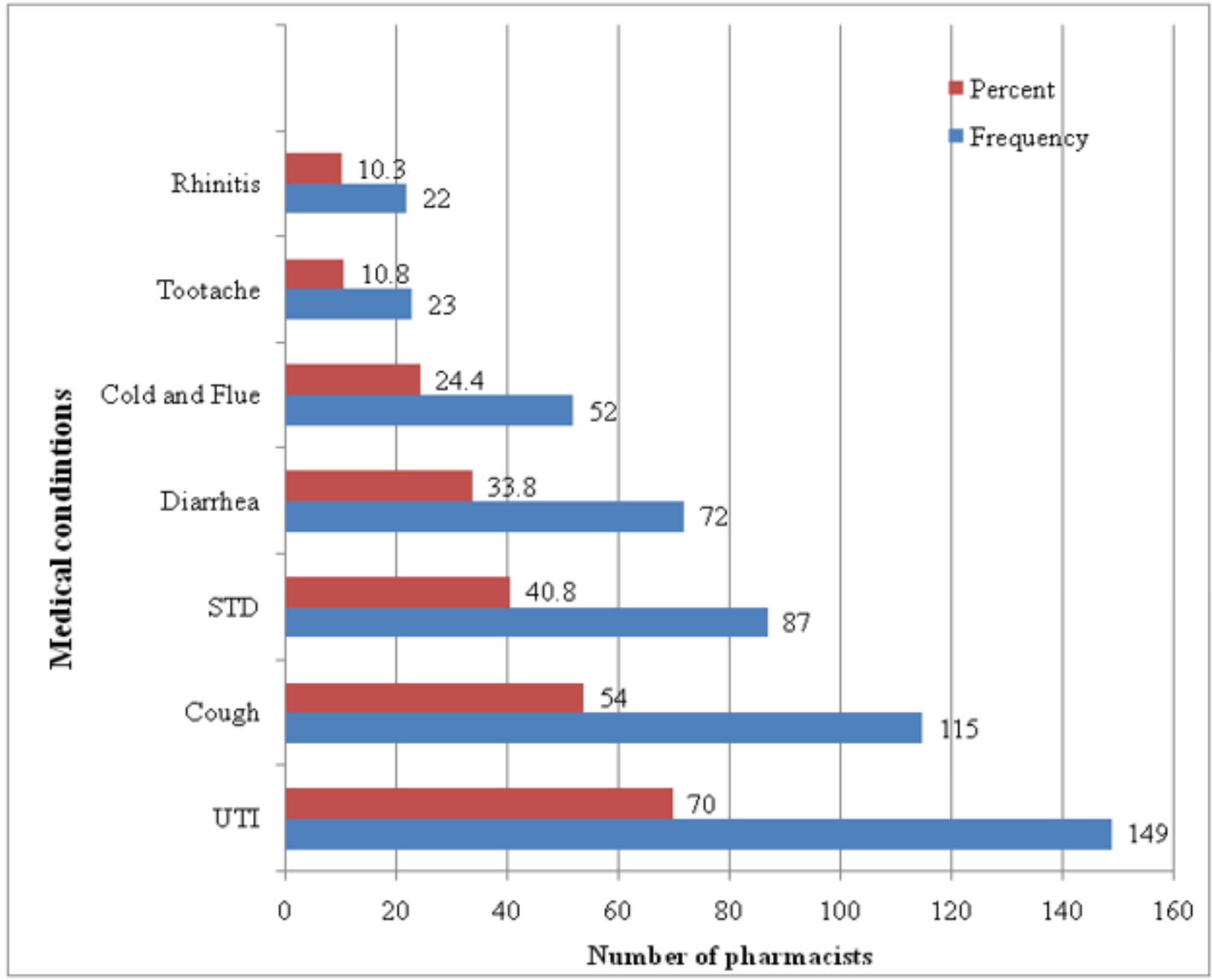

\section{Figure 4}

Medical conditions to which antibiotics are commonly dispensed without prescription as suggested by pharmacists 